Thus, the series expression for the potential on the axis $(y=0)$, derived in the same manner as the series expression for the potential of the vertical deflection field, leads to

$$
\begin{aligned}
\Phi^{\prime} & =\frac{2}{d}\left(V_{2}-V_{1}\right) \sum_{n=0}^{\infty}(-1)^{n} \exp \{\mp(2 n+1) \pi x / d\} x \gtrless 0 \\
& =\frac{2}{d}\left(V_{2}-V_{1}\right) e^{\pi x / d} /\left(1+e^{2 \pi x / d}\right) \\
& =(1 / d)\left(V_{2}-V_{1}\right) \operatorname{sech}(\pi x / d) .
\end{aligned}
$$

The focusing properties of this lens are obtained by integrating the paraxial equation ${ }^{9}$

$$
y^{\prime \prime}=-\frac{\Phi^{\prime} y+\Phi^{\prime \prime} y}{2 \Phi} .
$$

${ }^{9}$ Zworykin, et al., op. cit., see (12.12) on p. 402. The expressions for the focal lengths and principal plane positions of a weak lens are obtained by integrating the path equation first with $y=$ const and then integrating it again, treating the deviation of $y$ from its initial value as a small quantity, found in the first integration.
For weak lenses $\left[\left(V_{2}-V_{1}\right) /\left(V_{2}+V_{1}\right) \ll 1\right]$ the imageand object-side focal lengths $f_{i}$ and $f_{0}$ and the distance $h$ of the principal plane from the junction plane (imageand object-side principal planes coincide approximately) are given by

$$
\begin{aligned}
f_{i} & =\left(\frac{V_{2}+V_{1}}{V_{2}-V_{1}}\right)^{2}\left(\frac{2 V_{2}}{V_{2}+V_{1}}\right)^{1 / 2} \frac{\pi d}{4} \\
f_{0} & =\left(\frac{V_{2}+V_{1}}{V_{2}-V_{1}}\right)^{2}\left(\frac{2 V_{1}}{V_{2}+V_{1}}\right)^{1 / 2} \frac{\pi d}{4} \\
h & =-\frac{V_{2}+V_{1}}{V_{2}-V_{1}} \frac{\pi d}{4} .
\end{aligned}
$$

\section{ACKNOWLEDGMENT}

The author gratefully acknowledges the encouragement received from Dr. D. W. Epstein, at whose suggestion the analysis was carried out, as well as the invaluable assistance of Dr. F. Edelman, who programmed the numerical problems and supervised the machine computation required.

\title{
Coupled-Cavity Traveling-Wave Parametric Amplifiers: Part I-Analysis*
}

\author{
M. R. CURRIE†, SENIOR MEMBER, IRE, AND R. W. GOULD , MEMBER, IRE
}

\begin{abstract}
Summary-A general class of traveling-wave parametric amplifiers based on coupled-cavity filter circuits is described. This type of amplifier is particularly suited to microwave frequencies and incorporates new features that overcome some severe difficulties associated with other circuit structures. Traveling-wave diode-type amplifiers having relatively wide bandwidths and great simplicity at $S$ band have already resulted from this approach (details of experiments will be described in Part II).

An analysis is presented which provides detailed information on operating characteristics, including the effects of terminal impedances, reflected waves, circuit loss, etc., and also leads to a simple physical picture of the cumulative interaction mechanism in terms of coupled-mode concepts. This physical picture is emphasized throughout this paper. Gain bandwidth considerations are discussed in terms of a fundamental "interaction-impedance" parameter.

Representative calculated curves show that unilateral gains of 12 to $15 \mathrm{db}$ over relatively wide bandwidths are attainable with as few as 4 to 6 diodes. Methods of increasing the gain through use of ferrites and special circuit techniques are proposed.
\end{abstract}

* Received by the IRE, May 3, 1960; revised manuscript received, August 19, 1960. This work was presented at the Seventeenth Annual Conf. on Electron Device Res., Mexico City, Mexico; June, 1959. Calif. † Hughes Res. Labs., A Division of Hughes Aircraft Co., Malibu, $\ddagger$ Calif. Inst. Tech., Pasadena, Calif.

\section{INTRODUCTION}

$\Lambda$ GREAT deal of attention currently is being given to increasing the bandwidth capabilities of solidstate parametric amplifiers by application of traveling-wave circuit techniques. In many respects, this situation is reminiscent of the evolution of broadband traveling-wave tubes from klystron amplifiers employing resonant cavity circuits. As will be shown in this paper, this analogy not only provides much useful conceptual information but also suggests a specific analytical and experimental approach which already has resulted in traveling-wave diode-type parametric amplifiers having relatively wide bandwidths and great simplicity at microwave frequencies.

Following the analysis of Tien and Suhl ${ }^{1,2}$ of parametric amplification in a uniformly distributed non-

${ }^{1} \mathrm{P} . \mathrm{K}$. Tien, "Parametric amplification and frequency mixing in propagating circuits," J. Appl. Phys, vol. 29, pp. 1347-1357; Sep* tember, 1958.

${ }_{2}^{2} \mathrm{P}$. K. Tien and $\mathrm{H}$. Suhl, "A traveling wave ferromagnetic amplifier," Proc. IRE, vol. 46, pp. 700-706; April, 1958. 
dispersive medium, Engelbrecht demonstrated an experimental amplifier in the UHF region which approximated Tien's model and resulted in excellent bandwidth performance. ${ }^{3}$ This amplifier was based on a uniform transmission line (coaxial TEM mode) in which the nonlinear capacitive diodes were placed very close together with respect to wavelength so as to approximate a uniform nonlinear medium. Although the diodes were individually tuned for optimum performance, the essential nondispersive nature of the TEM mode was not changed.

In attempting to extrapolate directly this uniform transmission line approach to the microwave frequency range ( $S$ band and above) difficulties have been encountered which probably can be attributed to several factors.

First, the relatively low "interaction impedance" of such structures (in terms of voltage developed across the diode per unit power flow) implies a low gain per diode. With a large number of diodes, any nonuniformity in their characteristics can seriously affect amplifier gain. The diode losses also attenuate the pump power as it propagates down the system, which further reduces the contribution to gain of successive diode stages.

Second, such circuits can propagate some of the higher-order frequency components generated by mixing of the signal and the pump. In such systems, as shown recently by Roe and Boyd, ${ }^{4}$ exponential gain at the signal frequency may not occur; rather, energy conversion to the propagating cross-product frequencies can take place with very little gain at the signal frequency.

Finally, at microwave frequencies, the lead inductance associated with the diode, together with its stray capacitance, can establish a self-resonance at or below the operating frequency of the amplifier. In this case, the voltage that is developed across the capacitive $p-n$ junction itself may be greatly reduced with consequent severe degradation of amplifier performance.

This paper is concerned with a class of iterative circuits for traveling-wave parametric amplifiers which overcomes the first two difficulties and alleviates the third. It permits the realization of simple high-gain, broad-band parametric amplifiers at microwave frequencies.

The basic model is shown schematically in Fig. 1. It consists of a chain of inductively coupled cavities; each cavity is loaded by a diode in the capacitive region, and the diodes are individually pumped from an external circuit with the pump phase arranged so as to simulate a traveling wave. Some of the advantages of this general type of circuit are as follows:

3 R. S. Engelbrecht, "A low-noise nonlinear reactance traveling wave amplifier," Proc. IRE, vol. 46, p. 1655; September, 1958.

${ }^{4}$ G. M. Roe and M. R. Boyd, "Parametric energy conversion in distributed systems," Proc. IRE, vol. 47, pp. 1213-1218; July, 1959.

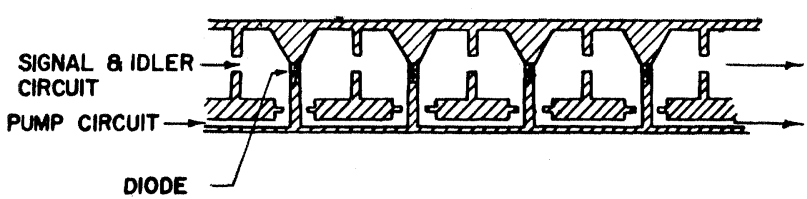

Fig. 1-Sketch of coupled-cavity type of traveling-wave parametric amplifier. Waves at the signal and idler frequencies propagate through a filter chain of inductively coupled cavities. Each cavity is loaded by a variable-capacitance diode at a position of maximum electric energy storage. Pump power is introduced from a parallel-feed system.

1) Since the structure constitutes a microwave bandpass filter circuit, the problems associated with the propagation of higher-frequency components are eliminated; these components can be made to fall in stop bands of the filter, where they cannot propagate and absorb energy. ${ }^{5}$

2) Unlike the case in which a uniform line is loaded by diodes, the diodes in this model are placed only in regions of concentrated electric energy storage. A higher voltage is developed across the nonlinear capacitance for a given power flow in the circuit. As a consequence, the gain per diode can be considerably higher than in the uniform case; the amplifier is greatly simplified by requiring fewer stages and lower pump power. As will be discussed, this situation can also be regarded from a more general viewpoint; a relation exists between gain per diode and bandwidth much like that between gain per unit length and bandwidth in traveling-wave tubes. By restricting the bandwidths to essentially the required value by means of a filter circuit, the gain per stage can be maximized.

3) The coupled-cavity circuit is particularly well adapted to microwave frequencies because it provides reasonably large diode spacings even at short wavelengths. For example, a direct scaling of Engelbrecht's amplifier, in which the diodes are spaced by $\frac{1}{8}$ wavelength at the signal frequency, would lead to prohibitively small spacings in the microwave region.

4) The circuit is a natural structure for incorporating ferrite elements as a possible means of improving stability by obtaining nonreciprocal attenuation. The regions of magnetic and electric energy storage are separated spatially so that elements sensitive to both electric and magnetic fields may be most effectively utilized in the amplifier design.

5) The use of parallel pumping permits efficient use of the available pump power and provides independent external adjustment of phase shift between sections so as to optimize gain, frequency response, and reverse attenuation.

6) The lead inductance of the diodes can be incorporated as part of the individual cavity circuits, thus

5 When the pumping is not too strong, the diode elastance varies essentially sinusoidally with time. It is only necessary then to suppress the next highest idler (sum of signal and pump frequencies) to eliminate the effects of all other idlers. 
alleviating some of the problems associated with selfresonance of the diode package.

The purpose of this paper is to describe the operation of filter-circuit traveling-wave parametric amplifiers of the type discussed above and to present an analysis that has proved very valuable in understanding some of their detailed characteristics. Certain aspects of this analysis are related to that of Bell and Wade ${ }^{6}$ for the case of uniform transmission lines which are periodically loaded by diodes. A companion paper presents results of an experimental program in which these ideas have been demonstrated and extended by adding several features not incorporated in the basic model.

We shall first discuss the properties of coupled-cavity circuits and give a qualitative picture of the conditions under which an idler wave, which closely couples to the signal wave and leads to exponential growth, can be generated. The subsequent analysis is based on an equivalent circuit that closely represents the characteristics of its coupled-cavity microwave analog. The analytical approach has the advantage of taking into account the effects of terminal impedances and reflected waves and loss, all of which are of first-order importance in amplifier design. Detailed results of computer calculations clearly illustrate the essential characteristics of this type of amplifier and point up certain basic similarities with traveling-wave tubes. Finally, this approach is related to that of Tien in terms of a fundamental new interaction-impedance parameter.

\section{Discussion of Model and Analysis}

An equivalent-circuit representation of the coupledcavity traveling-wave parametric amplifier is shown in Fig. 2. This particular circuit was chosen for analysis because it has been successfully applied in studying similar problems for traveling-wave-tube amplifiers. ${ }^{7}$ In both cases, the basic cavity circuit elements are designed so as to concentrate the electric-energy storage at the point of active interaction (whether the interaction occurs with a time-varying capacitance or with an electron beam) and the cavities are inductively coupled. For fractional bandwidths less than about 25 per cent, this equivalent circuit can provide an accurate quantitative description of the characteristics of the actual distributed microwave circuit. This has been established by extensive measurements. For increasing bandwidths, the quantitative accuracy is not as good because of the use of frequency-independent elements in the equivalent circuit. However, the representation contains all the essentials of the physical problem, and the results of the analysis apply in a qualitative way to

${ }^{6} \mathrm{C}$. V. Bell and G. Wade, "Circuit considerations in traveling wave parametric amplifiers," 1959 IRE WESCON Convention RECORD, pt. 2, pp. 75-82.

${ }^{7}$ R. W. Gould, "Characteristics of traveling wave tubes with periodic circuits," IRE Trans. ON Electron Devices, vol. ED-5, pp. 186-195; July, 1958. other possible iterated circuits of thc general type indicated in Fig. 1.

In discussing the model, it is useful to define the following dimensionless parameters:

$$
\lambda=\omega \sqrt{L C_{0}}
$$

is frequency normalized to the midband frequency $\left(L C_{\theta}\right)^{-1 / 2}$ of the cold filter circuit;

$$
k=M / L
$$

is the coefficient of coupling between cavities;

$$
q=R \sqrt{\frac{C_{0}}{L}}
$$

is the reciprocal resonator $Q$ and provides a measure of resistive circuit loss. (Note that $C_{o}=1 / S_{0^{\circ}}$ )

The general properties of the model are indicated in the Brillouin diagram of Fig. 3. We will use this representation throughout as a convenient means for studying the properties of amplifiers of the filter-circuit type. Here we have assumed that $k$ is positive. For negative $k$, the fundamental branch would be a backward wave, but this would not alter the nature of the problem. The abscissa $\theta$ is the phase shift per resonator corresponding to a dependence

$$
i_{n}=i_{n-1} \exp (-\alpha-j \theta)
$$

where $\alpha$ is a measure of the circuit loss. The upper and

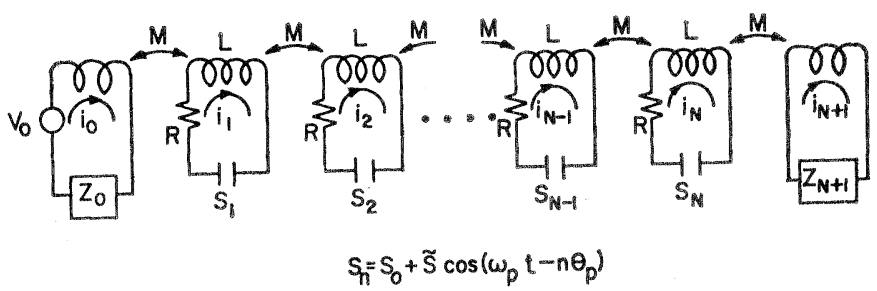

Fig. 2-Equivalent circuit of coupled-cavity traveling-wave parametric amplifier including arbitrary terminal impedances. The time variation of elastance of the $n$th cavity is indicated; $S_{o}=1 / C_{0}$, where $C_{o}$ is the combined localized static capacitance of cavity and diode.

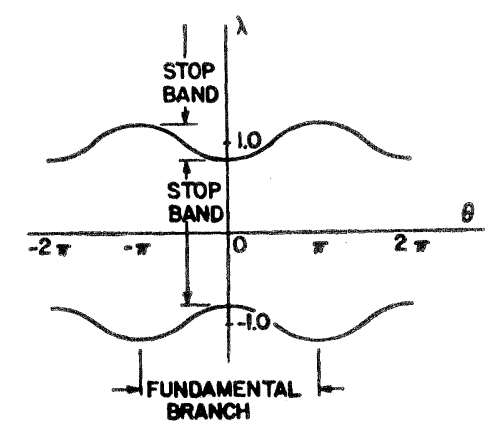

Fig. 3-Brillouin diagram of coupled-cavity filter circuit; $\lambda$ is normalized frequency, $\theta$ is phase shift per section. Both the positive and negative frequency passbands are shown. 
lower cutoff frequencies of the pass band are given by

$$
\lambda_{\text {lower }}^{\text {upper }}=\frac{1}{\sqrt{1 \mp 2 k}} \cong 1 \pm k \quad(k \ll 1),
$$

so that the fractional bandwidth is approximately equal to $2 k$ for $k$ small. The pass band shown in Fig. 3 represents only the principal pass band of the actual microwave filter circuit in which both the signal and idler waves propagate. Other higher-frequency bands will exist depending upon the specific geometry; these can be adjusted so that a frequency equal to the sum of the signal and pump frequencies falls in a stop band, thus eliminating the existence of all higher-frequency mixing components. (As discussed in Part II, the existence of a pass band at the sum frequency can even be used to advantage if it can be made to have a certain dispersion characteristic.) The filter characteristic as shown on the Brillouin diagram is periodic in $\theta$ with a period of $2 \pi$. In the case of a microwave filter circuit, this periodicity represents the space-harmonic structure due to spatial periodicity of the fields. In parametric amplifiers, however, only the fundamental branch $(-\pi<\theta<\pi)$ is of importance.

In the case of no resonator loss $(q=0)$, there is no attenuation in the pass band. For nonzero $q$, the midband attenuation is

$$
\alpha=\frac{q}{2 k}
$$

nepers per resonator. Outside of the pass band, of course, only evanescent waves can exist.

For any specific coupled-cavity circuit, the lumped parameters of the equivalent circuit can be determined directly from measurements of midband frequency, bandwidth, capacitance, and loss. The capacitance is obtained from an impedance measurement and, for reasonably high resonator and diode $Q$, is equal to the sum of diode and cavity capacitance.

We begin the analysis by writing Kirchhoff's voltage law for the $n$th resonant circuit of Fig. 2:

$L \frac{d^{2} q_{n}}{d t^{2}}+M \frac{d^{2} q_{n-1}}{d t^{2}}+M \frac{d^{2} q_{n+1}}{d t^{2}}+R \frac{d q_{n}}{d t}+S_{n} q_{n}=0$,

where $q_{n}$ is the charge on the $n$th capacitor. The current in the $n$th resonator is $i_{n}=d q_{n} / d t$. It will be assumed that the excitation at the pump frequency causes the elastance $S_{n}$ to vary as ${ }^{8}$

$$
\begin{aligned}
S_{n} & =S_{0}+2 \tilde{S} \cos \left(\omega_{p} t-n \theta_{p}\right) \\
& =S_{0}+\tilde{S}\left[e^{j\left(\omega_{p} t-n \theta_{p}\right)}+e^{-j\left(\omega_{p} t-n \theta_{p}\right)}\right] .
\end{aligned}
$$

8 In general, the time variation of elastance must be expressed as a Fourier series of terms varying at harmonics of the pump frequency $\omega_{p}$. Assuming $S_{n}$ to be of the form (8) does not restrict the analysis to situations in which these terms are of negligible magnitude. It merely implies that frequency components generated as a result of mixing between the signal and the harmonic pump terms cannot propagate in the filter circuit and enter in any significant way in the interaction process.
It is seen that a time-varying elastance of this form generates new frequencies $\omega_{1} \pm \omega_{p}$ from a signal at frequency $\omega_{1}$. Only if these new frequencies can propagate through the chain of coupled resonators with the proper phase velocity can the contributions from adjacent cavities be cumulative. We specify that the frequency $\omega_{1}+\omega_{p}$ fall in a stop band, and thus we can neglect this frequency as well as all higher idlers. We retain only the frequency $\omega_{2}=\omega_{1}-\omega_{p}$, which, if $\omega_{p}$ is in the vicinity of twice the signal frequency, can propagate in the negative frequency pass band. ${ }^{9}$

Let

$$
q_{n}=A_{n} e^{j \omega_{1} t}+B_{n} e^{j \omega_{2} t} .
$$

Upon substituting (9) and (8) into (7) and requiring that coefficients of $e^{j \omega_{1} t}$ and $e^{j \omega_{2} t}$ vanish separately, we obtain

$$
\begin{aligned}
-\lambda_{1}^{2}\left[A_{n}+k A_{n-1}+\right. & \left.k A_{n+1}\right] \\
& +j \lambda_{1} q A_{n}+A_{n}+\epsilon B_{n} e^{-j n \theta_{p}}=0
\end{aligned}
$$

and

$$
\begin{aligned}
-\lambda_{2}{ }^{2}\left[B_{n}+k B_{n-1}+\right. & \left.k B_{n+1}\right] \\
& +j \lambda_{2} q B_{n}+B_{n}+\epsilon A_{n} e^{j n \theta_{p}}=0,
\end{aligned}
$$

where $\lambda_{1}$ and $\lambda_{2}$ are the normalized signal and idler frequencies, respectively, and $\epsilon$ is a pump parameter,

$$
\epsilon=\tilde{S} / S_{0}
$$

Since amplitudes of other frequency components (higher idlers) are assumed to be vanishingly small in this model, we do not write their governing equations.

When there is no pump $(\epsilon=0),(10)$ and (11) reduce to separate characteristic equations for propagation of the signal frequency $\lambda_{1}$ and the idler frequency $\lambda_{2}$. For a dependence of the form (4), this characteristic equation is

$$
-\lambda^{2}(1+2 k \cos \theta)+1=0,
$$

and its solution is the Brillouin diagram in Fig. 3.

The effect of the additional terms in (10) and (11) arising from the pump is to couple the signal and idler equations and, in effect, to couple the modes propagating at the signal and idler frequencies. It should be kept in mind that the idler frequency $\lambda_{2}$ would not actually exist in the absence of the pump, although a disturbance at this frequency could propagate through the resonators, if excited. Because of the time-varying capacitance, a disturbance at frequency $\lambda_{2}$ has associated with it a disturbance at $\lambda_{1}$ and vice versa.

Eqs. (10) and (11) comprise a set of linear difference equations with nonconstant coefficients (the last coefficient in each equation depends on $n$ ). By making

\footnotetext{
${ }^{9}$ It is convenient in this analysis to employ the notion of negative frequency and thus avoid the necessity of using cumbersome complex conjugate quantities. Physical quantities are given by the real parts of the expressions thus obtained.
} 
the substitution $B_{n}=B^{\prime}{ }_{n} e^{j n \theta p}$, however, a set of constant coefficient equations is obtained:

$$
\begin{aligned}
-\lambda_{1}{ }^{2}\left[A_{n}+k A_{n-1}+k A_{n+1}\right] & \\
& +j q \lambda_{1} A_{n}+A_{n}+\epsilon B^{\prime}{ }_{n}=0 \\
-\lambda_{2}{ }^{2}\left[B^{\prime}{ }_{n}+k e^{-j \theta_{p}} B^{\prime}{ }_{n-1}\right. & \left.+k e^{+j \theta_{p}} B^{\prime}{ }_{n+1}\right] \\
& +j q \lambda_{2} B_{n}^{\prime}+B_{n}^{\prime}+A_{n}=0 .
\end{aligned}
$$

We interpret $B^{\prime}{ }_{n}$ as follows. A signal at $\omega_{2}$ would be described by $q_{n}=e^{j \omega_{2} t} B_{n}$. The time-varying capacitor (8) generates a voltage at frequency $\omega_{1}$ given by

$$
\widetilde{S} e^{j \omega_{1} t} B_{n} e^{-j n \theta_{p}}=\widetilde{S} e^{j \omega_{1} t} B^{\prime}{ }_{n} .
$$

Thus the variation of the phase of this converted signal is contained in the factor $B^{\prime}{ }_{n}$. In the limit of a very weak pump, $B^{\prime}{ }_{n} \sim e^{-j n\left(\theta_{2}+\theta_{p}\right)}$, where $\theta_{2}$ is the phase shift of the circuit at $\omega_{2}$.

To solve (14) and (15), we assume the solution to be of the form $A_{n}=A_{0} \mu^{n}, B^{\prime}{ }_{n}=B^{\prime}{ }_{\nu} \mu^{n}$, where $\mu$ is a complex constant. In the earlier discussion of the characteristics of the cold circuit, we let $\mu=e^{-(\alpha+j \theta)}$, where $\alpha$ and $\theta$ are the attenuation and phase shift per section, respectively. For a solution, $\mu$ must satisfy the determinantal equation

$$
\begin{aligned}
& {\left[-\lambda_{1}{ }^{2}\left(1+\frac{k}{\mu}+k \mu\right)+j q \lambda_{1}+1\right]} \\
& \quad\left[-\lambda_{2}{ }^{2}\left(1+\frac{k}{\mu e^{j \theta_{p}}}+k \mu e^{j \theta_{p}}\right)+j q \lambda_{2}+1\right]-\epsilon^{2}=0 .
\end{aligned}
$$

For each value of $\lambda_{1}$, this equation is satisfied by four different values of $\mu$. The solutions for $q=0, \epsilon=0$ are particularly simple and have been previously discussed. The first factor in (16) leads to the usual dispersion curve, which is repeated as the solid curve in Fig. 4. The second factor has the same form as the first if we write $\mu=\mu^{\prime} e^{j \theta p}$. Thus, a curve of $\lambda_{2}$ vs $\theta^{\prime}$ is identical in form to the solid curves of Fig. 4.

It is useful to express the latter solution in terms of $\lambda_{1}$ and $\theta$. Noting that $\lambda_{1}=\lambda_{2}+\lambda_{p}$ and $\theta=\theta^{\prime}+\theta_{p}$, we can construct the second set of solutions from the first by simply displacing the solid curve of Fig. 4 upward by an amount $\lambda_{p}$ and to the right by an amount $\theta_{p}$. This results in the dashed curves shown.

We are now in a position to give a simple physical picture of the traveling-wave interaction mechanism and an interpretation of this analysis. We may regard the dashed curves in Fig. 4 as giving the phase shift per section of a disturbance at $\lambda_{1}$, which would result from the conversion of a signal at $\lambda_{2}$ by a very weak pump ( $\epsilon$ small). If this phase shift coincides with the normal phase of the circuit at $\lambda_{1}$ (as given by the solid curve), then the converted signal can propagate along the circuit in phase with the signal at $\lambda_{1}$ and a cumulative effect from section to section will exist. We expect this effect to be strongest and the gain to be maximum

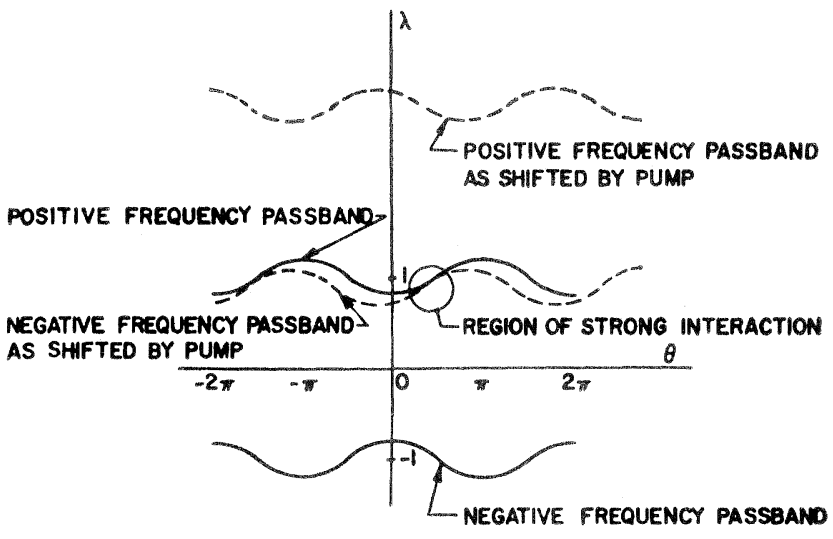

Fig. 4-Generation of the "idler curve," showing the phase shift per section of a wave at $\lambda_{1}$, which results from conversion of a wave at $\lambda_{2}$ (idler frequency) by mixing with a weak pump. The idler curve is generated by translating the negative frequency dispersion curve to the right by a distance $\theta_{p}$ and vertically upward by $\lambda_{p}$. A higher-order idler curve is also shown; it falls in a stop band of the filter circuit.

when the solid and dashed curves of Fig. 4 coincide. At this point

$$
\theta\left(\lambda_{2}\right)+\theta_{p}=\theta\left(\lambda_{1}\right)
$$

where $\lambda_{2}=\lambda_{1}-\lambda_{p}$. This is just the relation found by Tien ${ }^{1}$ except for the change in the signs preceding $\lambda_{2}$ and $\theta\left(\lambda_{2}\right)$, which arises from our use of negative frequencies.

The range of frequency over which the solid and dashed lines of Fig. 4 nearly coincide determines the bandwidth over which traveling-wave parametric amplification can be obtained. The difference in phase shifts that can be tolerated and still have amplification increases as the pump parameter $\epsilon$ is increased. This will be illustrated in detail later.

The above description bears a close analogy to the theory of coupling of modes of propagation ${ }^{10,11}$ and, as will be discussed in the next section, has proved to be very useful in understanding some of the detailed characteristics of iterated traveling-wave parametric amplifiers as they result from the present analysis.

A complete solution of the problem is given by a superposition of the four wave solutions; i.e.,

$$
q_{n}=\sum_{r=1}^{4} A_{o r} \mu_{r}{ }^{n} e^{j \omega_{1} t}+\sum_{r=1}^{4} B_{o r}^{\prime} e^{j n \theta_{p}} \mu_{r}{ }^{n} e^{j \omega_{2} t}
$$

gives the charge on the $n$th capacitor. The four complex roots of the determinantal equation are $\mu_{1} \cdots \mu_{4}$, and $A_{01} \cdots A_{04}$ are arbitrary constants, which are determined by the boundary conditions of the model; $B_{o r}^{\prime}$ is related to $A_{\text {or }}$ by means of (14). The current in the $n$th

${ }^{10} \mathrm{~J}$. R. Pierce, "Coupling of modes of propagation," J. Appl. Phys., vol. 25, p. 179; February, 1954.

11 J. R. Pierce and P. K. Tien, "Coupling of modes in helices," Proc. IRE, vol. 42, p. 1389; September, 1954. 
resonator circuit is therefore given by

$$
\begin{aligned}
i_{n}=\sum_{r=1}^{4} I_{o r} \mu_{r}{ }^{n} & \left\{e^{j \omega_{1} t}-e^{j \omega_{2} t} \frac{\lambda_{2}}{\epsilon \lambda_{1}} e^{j n \theta_{p}}\right. \\
\cdot & {\left.\left[1+j q \lambda_{1}-\lambda_{1}{ }^{2}\left(1+\frac{k}{\mu_{r}}+k \mu_{r}\right)\right]\right\}, }
\end{aligned}
$$

where we have written $I_{\text {or }}=j \omega_{1} A_{\text {or }}$. The arbitrary constants $I_{01} \cdots I_{04}$ are determined from the boundary conditions, which are written as separate equations for the input and output circuits:

$$
\begin{aligned}
& M \frac{d i_{1}}{d t}+i_{o} Z_{o}=V_{o} e^{j \omega_{1} t}, \\
& M \frac{d i_{N}}{d t}+i_{N+1} Z_{N+1}=0 .
\end{aligned}
$$

Substituting (19) into (20) and (21) and equating $\omega_{1}$ terms and $\omega_{2}$ terms separately, we obtain four equations in the four unknowns:

$$
\begin{gathered}
\sum_{r=1}^{4} I_{o r}\left[j \lambda_{1} k \mu_{r}+Z_{o}^{o}\left(\lambda_{1}\right)\right]=V_{o}^{o} \\
\sum_{r=1}^{4} I_{o r} \mu_{r}^{N}\left[j \lambda_{1} k+\mu_{r} Z_{N+1}^{o}\left(\lambda_{1}\right)\right]=0 \\
\sum_{r=1}^{4} I_{o r}\left[1+j q \lambda_{1}-\lambda_{1}{ }^{2}\left(1+k \mu_{r}+\frac{k}{\mu_{r}}\right)\right] \\
\cdot\left[j \lambda_{2} k \mu_{r} e^{j \theta_{p}}+Z_{o}^{o}\left(\lambda_{2}\right)\right]=0 \\
\sum_{r=1}^{4} I_{o r} \mu_{r}^{N}\left[1+j q \lambda_{1}-\lambda_{1}{ }^{2}\left(1+k \mu_{r}+\frac{k}{\mu_{r}}\right)\right] \\
\cdot\left[j \lambda_{2} k+Z_{N+1}^{o}\left(\lambda_{2}\right) \mu_{r} e^{j \theta_{p}}\right]=0
\end{gathered}
$$

where we have written the result directly in terms of normalized variables using the additional definitions

$$
\begin{gathered}
Z_{o}^{o}(\lambda)=\sqrt{\frac{\overline{C_{o}}}{L}} Z_{o}(\omega), \quad Z_{N+1}^{o}(\lambda)=\sqrt{\frac{\bar{C}_{o}}{L}} Z_{N+1}(\omega), \\
V_{o}^{o}=\sqrt{\frac{C_{o}}{L}} V_{o} .
\end{gathered}
$$

Results, which are discussed in the next section, are obtained as follows. The constants $k, q$, and $N$ are determined from the fractional bandwidth, midband attenuation, and number of elements of the particular filter circuit being considered; similarly, the frequencydependent terminating impedances $Z_{0}^{0}(\lambda)$ and $Z_{N+1}^{o}(\lambda)$ are determined from the input and output matching configurations. The pump strength parameter $\boldsymbol{\epsilon}$ defined by (12) is related to the total swing in capacitance approximately by

$$
\epsilon=\frac{1}{4} \frac{\Delta S}{S_{o}} \cong \frac{1}{4} \frac{\Delta C}{C_{o}},
$$

where $\Delta C$ is a function of pump voltage, bias, and the particular capacitance-voltage characteristic of the diodes considered. $\lambda_{p}$ and $\theta_{p}$, the pump frequency and phase shift between sections, respectively, exert a major influence on the amplifier characteristics; the choice of these parameters will be discussed presently. With the above parameters established, the determinantal equation (16) is solved for the four values of $\mu$ and as a function of the signal frequency $\lambda_{1}$. Eqs. (22) through (25) are then solved for the wave amplitudes $I_{o}$, with $V_{o}^{o}=1$ and the current in the input and output circuits computed from (19).

Computations were made on an IBM 704 digital computer. As a check on the accuracy of the results, it was verified that the terminal characteristics in each case (for zero circuit loss) are in agreement with the ManleyRowe relations. ${ }^{12}$ That is, it was determined that $P_{1} / \lambda_{1}=P_{2} / \lambda_{2}$, where $P_{1}$ is the sum of the powers appearing in the input and output circuits at frequency $\lambda_{1}$, and $P_{2}$ is the total power at $\lambda_{2}$.

\section{Results of ANALysis}

Since the gain, bandwidth, and stability depend sensitively upon $\lambda_{p}$ and $\theta_{p}$, let us first develop a qualitative picture of how these parameters affect amplifier performance. This has proved to be of considerable value in arriving at optimum adjustments of experimental models in the laboratory. In the foregoing section, we discussed the generation of a propagating wave at the idler frequency and how this idler response can be represented on a Brillouin diagram (Fig. 4). The advantage of constructing an "idler curve" in this manner is that it relates the cumulative traveling-wave parametric-amplification mechanism to familiar concepts of coupled modes. When the "signal" and "idler" curves of Fig. 4 are close together, the phase conditions are favorable for cumulative interaction; the modes, which carry power in the same direction, can couple to establish a pair of exponentially growing and decaying waves. Indeed, the condition for maximum coupling from this point of view has been shown to coincide with the optimum relation between phase velocities found by Tien. Extending this picture slightly, we can develop a feeling for how $\lambda_{p}$ and $\theta_{p}$ affect gain-bandwidth, band-edge response, and stability and thus arrive at criteria for choosing and adjusting these parameters.

${ }^{12}$ J. M. Manley and H. E. Rowe, "Some general properties of nonlinear elements-Part I. General energy relations," PROC. IRE, vol. 44, pp. 904-913; 1956. 
Consider several specific situations. First, let us assume that $\lambda_{p}=2$ (pump frequency equal to twice the midband frequency of the filter circuit) and $\theta_{p}=\pi$. In this case, the idler curve coincides with the signal curve, as indicated in Fig. 5(a). The condition for maximum gain (17) is satisfied here for every frequency within the pass band of the filter circuit. Notice, however, that this optimum condition is also fulfilled for waves traveling in the reverse direction, as indicated by the left-hand or negative phase-shift branch of the diagram where the group velocity $\left(v_{g} \sim d \lambda / d \theta\right)$ is negative. Thus, the amplifier is bilateral and in the absence of perfect terminations would be unstable.

Now let $\lambda_{p}$ become somewhat less than 2 , but main$\operatorname{tain} \theta_{p}=\pi$. As shown in Fig. 5(b), this results in a downward displacement of the idler curve. The gain, although reduced, is still bilateral; i.e., for propagation in either the forward or backward direction, the horizontal spacing of the curves is identical. Notice also that the maximum bandwidth over which amplification can now take place (i.e., the bandwidth in which waves can propagate at both the signal frequency $\lambda_{1}$ and idler frequency $\left.\left|\lambda_{2}\right|=\left|\lambda_{1}-\lambda_{p}\right|\right)$ is measured by the vertical overlap of the signal and idler curves and is reduced below the bandwidth of the cold $(\epsilon=0)$ filter circuit.

Finally, let $\lambda_{p}<2, \theta_{p}<\pi$, as shown in Fig. 5(c). Since the idler curve is thus displaced horizontally, the gain will be different in the forward and backward directions. For the case shown, almost unilateral gain in the forward direction is possible, hence stability. Exactly how close the curves can be brought together in Fig. 5(c) depends on still another factor, viz., band-edge response. As will be shown later, the interaction impedance at the band edge is higher than that in the center of the band and severe reflections at the terminations occur here. Thus, in this qualitative picture, unless a certain departure from synchronism (i.e., intercurve spacing) is maintained at the band edge, the amplifier may oscillate at that point. This minimum spacing (which is a function of pump strength) determines how much gain can be achieved at the band center.

We have thus illustrated the important factors which determine the choice of $\lambda_{p}$ and $\theta_{p}$ and establish limits on the performance of this type of amplifier. We would like to achieve the objectives of high unilateral gain with maximum utilization of the filter's cold bandwidth. Unilaterality can be achieved by making $\theta_{p}<\pi, \lambda_{p}<2$, thus reducing the maximum possible bandwidth of amplification. Gain is limited by band-edge stability considerations. To optimize performance in the presence of these conflicting requirements, $\lambda_{p}$ and $\theta_{p}$ must be carefully balanced. (Note that the condition $\theta_{p}<\pi, \lambda_{p}<2$ leads to utilization of the lower part of the filter pass band. It can be similarly shown that the condition $\theta_{p}>\pi, \lambda_{p}>2$ leads to utilization of the upper part of the pass band consistent with unilateral amplification; however, only the former mode of operation is discussed in this paper for convenience in the presentation of re-

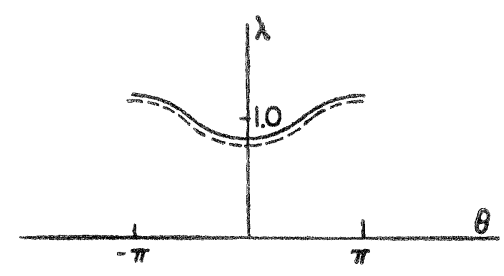

(a)

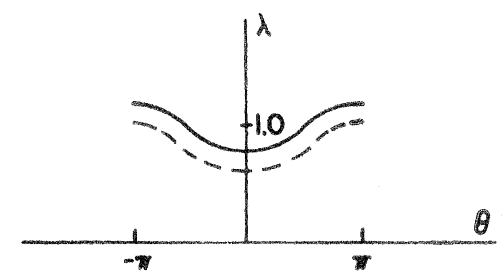

(b)

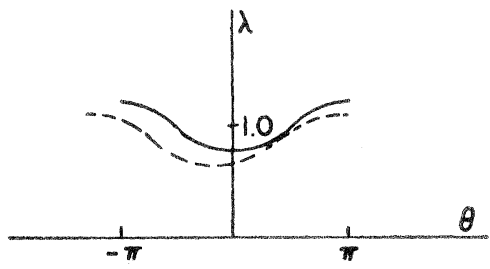

(c)

Fig. 5-With the use of coupled-mode ideas, these curves illustrate how pump frequency $\lambda_{p}$ and pump phase $\theta_{p}$ affect amplifier performance. The gain (i.e., coupling) decreases as the signal and idler curves are moved apart. (a) $\lambda_{p}=2, \theta_{p}=\pi$; (b) $\lambda_{p}<2, \theta_{p}=\pi$; (c) $\lambda_{p}<2, \theta_{p}<\pi$.

sults.) The above discussion assumes a symmetrical dispersion curve; as will be shown in Part II, asymmetry in the dispersion curve can remove the condition on $\lambda_{p}$.

Fig. 6 illustrates the typical nature of the solutions of the characteristic equation (16). The uncoupled signal and idler curves are shown in Fig. 6(a) with the phase characteristics of the coupled system [i.e., roots of (16)] plotted in Fig. 6(b). There are four waves at each frequency. A solid line indicates a wave of constant amplitude for which $\alpha=0$ where $|\mu|=e^{\alpha}$. It is thus seen that the waves in the reverse direction are virtually unaffected by the pump signal. On the other hand, the signal and idler waves in the forward direction are coupled strongly to form a pair of waves (at each frequency) that are growing and decaying; this is indicated by the dashed line for which the solutions have equal and opposite values of $\alpha$. In traveling-wave parametric amplifiers, it is the exponentially growing wave in which we are interested.

The study of the normal modes of the system can provide valuable insight into the behavior of the traveling-wave amplifiers. However, it is also necessary to calculate the extent to which each of the normal modes is excited in order to compute the over-all amplifier gain. This is given by solution of (22) through (25) for the mode amplitudes, from which the current in the output circuit at the signal frequency can be obtained. The power gain is defined as

$$
G=\frac{\text { power in load }}{\text { power available at input }}=\frac{\left|I_{N+1}\right|^{2} R_{N+1}}{V_{o}^{2} / 4 R_{o}},
$$




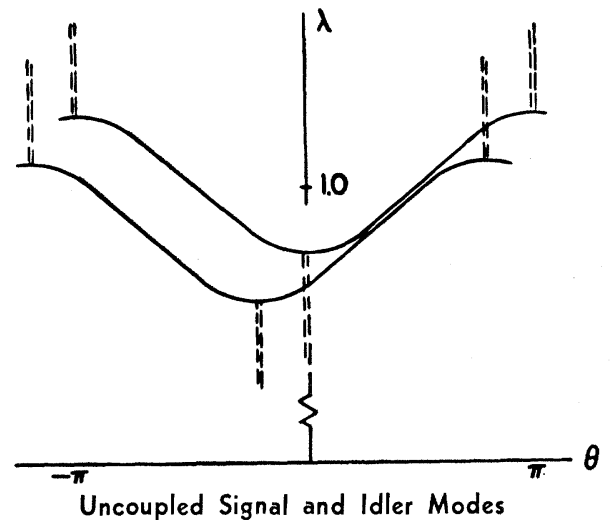

(a)

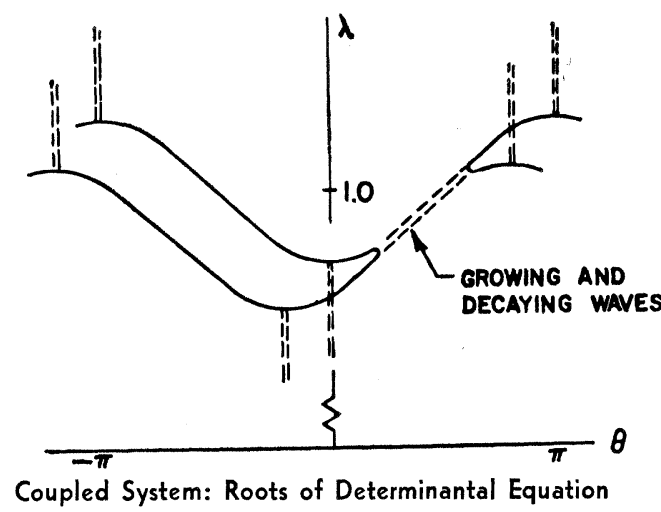

(b)

Fig. 6-Nature of the solutions of the determinantal equation. The signal and idler modes in the upper diagram are coupled by the pump as shown in the lower diagram. Solid lines indicate waves of constant amplitude; dashed lines indicate a conjugate pair of growing and decaying waves.

or, in terms of our normalized parameters,

$$
G=4\left|I_{N+1}\right|^{2} R_{o}^{o} R_{N+1}^{o}
$$

with the input voltage $V_{o}^{o}$ normalized to unity. In this formulation, the gain includes the effect of reflections from both input and output terminations.

In Figs. 7-9 (next page), we have presented calculated curves of gain vs frequency. These curves are not intended to represent optimized performance; rather, they have been chosen to illustrate typical effects of varying the principal parameters pump frequency $\lambda_{p}$, pump phase shift between sections $\theta_{p}$, and pump strength $\epsilon$. For convenience, the fractional bandwidth of the cold filter circuit has been chosen to be 10 per cent $(k=0.05)$. As shown in Appendix I, these results can be scaled to other bandwidths by simple scaling rules. The loss factor $q$ is 0.002 , corresponding to a cold insertion loss that is approximately $0.2 \mathrm{db}$ at midband and rising to about $4 \mathrm{db}$ per section in the neighborhood of the band edges. This corresponds roughly to the values measured in experimental models. The calculations are for a six-section amplifier; extensions to other numbers of active elements will be considered later.

Fig. 7 illustrates the effects of varying pump frequency $\lambda_{p}$ while maintaining pump phase $\theta_{p}$ and amplitude $\epsilon$ constant at the values 2.85 radians and 0.033 , respectively. The curves are seen to be approximately symmetrical about the degenerate frequency $\lambda_{p} / 2$. As $\lambda_{p}$ is reduced, several things occur: midband gain decreases, bandwidth decreases, and ripples in gain are smaller, particularly at the band edges.

This behavior follows immediately from our previous discussion. The present situation qualitatively resembles that shown in Fig. 5(c) or Fig. 6(a). As $\lambda_{p}$ decreases, the idler curve is displaced vertically downward from the signal curve. The maximum bandwidth over which both signal and idler frequencies can propagate is thus reduced. In this case, a maximum utilization of about 80 per cent of the total bandwidth of the filter circuit is shown for $\lambda_{p}=1.97$. Also, with decreasing $\lambda_{p}$, the separation between signal and idler curves increases; i.e., the signal and idler wave velocities depart more and more from synchronism, resulting in smaller midband gain. Similarly, the gain ripples are reduced in magnitude, because of a reduced gain in the backward direction, which makes the amplifier less sensitive to reflections at the output termination, and because, at the lower cutoff frequency, the departure from the synchronous condition begins to exceed the value above which signal and idler waves are essentially uncoupled.

At reasonably high gains, the Manley-Rowe relations $^{12}$ show that in the absence of loss, the amount of output power at the signal and idler frequencies is about equal. Therefore, the signal and idler waves essentially interchange roles as the signal passes through the degenerate point and the response is more or less symmetrical about the half-pump frequency. Over-all bandwidth cannot exceed twice the difference between $\lambda_{p} / 2$ and the nearer filter cutoff frequency.

Fig. 8 shows the effect of varying the pump phase shift. In terms of the Brillouin diagram, increasing $\theta_{p}$ corresponds to displacing the idler curve horizontally to the right. In this case, the bandwidth remains approximately constant (i.e., the normalized degenerate frequency is fixed at 0.98 ). For smaller values of $\theta_{p}$, in which midband synchronism is most nearly approached, the general gain level is the highest, although with large variations across the band in this particular case because of poor amplifier terminations.

Finally, in Fig. 9 it is seen that increased pump power results in larger gain, as expected, and also in a slightly enhanced bandwidth. The latter is due to the fact that the reduction in signal-idler coupling in the vicinity of the band edge, which results from departure from synchronism, is compensated by increased capacitance variation (i.e., pump power).

Although the terminations can be greatly improved so as to smooth out the gain peaks (see below), the band edge poses a special problem. This can be seen by introducing the concept of an "interaction impedance," which measures the quality of a particular coupledcavity circuit design. Letting $P$ represent the average power flow associated with a peak voltage $V_{n}$ across the localized capacitance of the $n$th cavity (loaded by a 


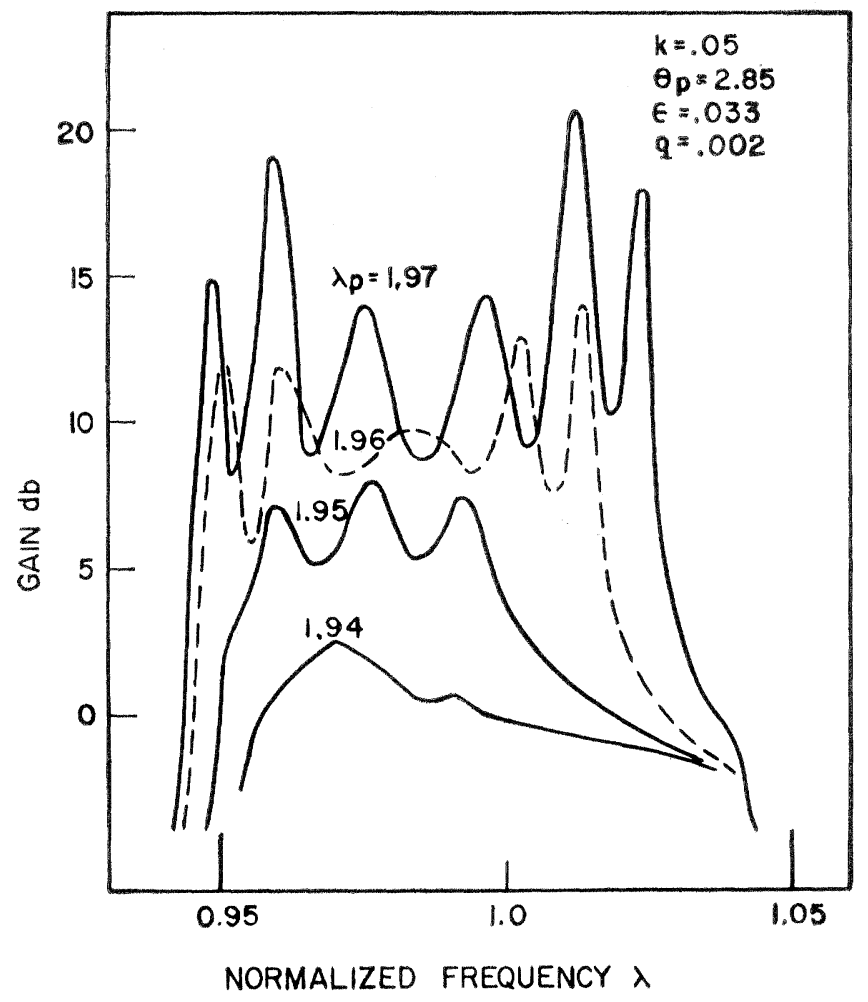

Fig. 7-Gain vs frequency for a six-section amplifier, showing the effect of varying pump frequency $\lambda_{p}$. A filter bandwidth of 10 per cent was chosen for convenience.

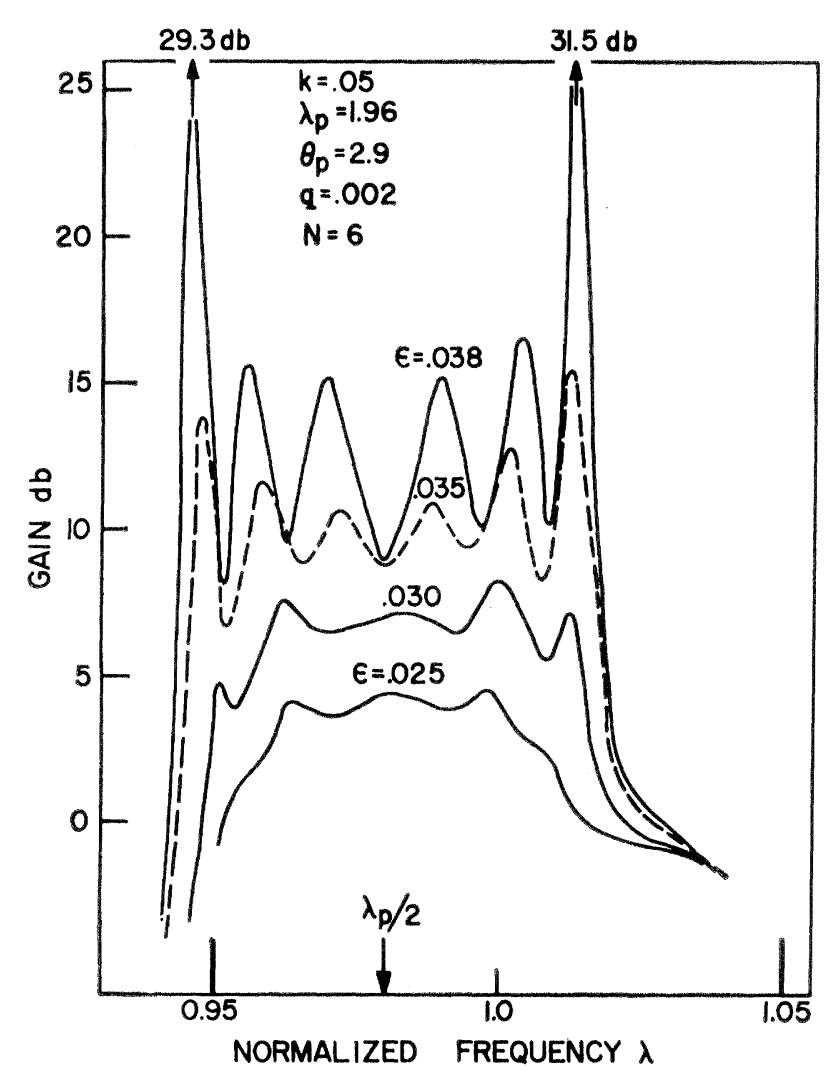

Fig. 9-Effect of increasing pump amplitude $\epsilon$.

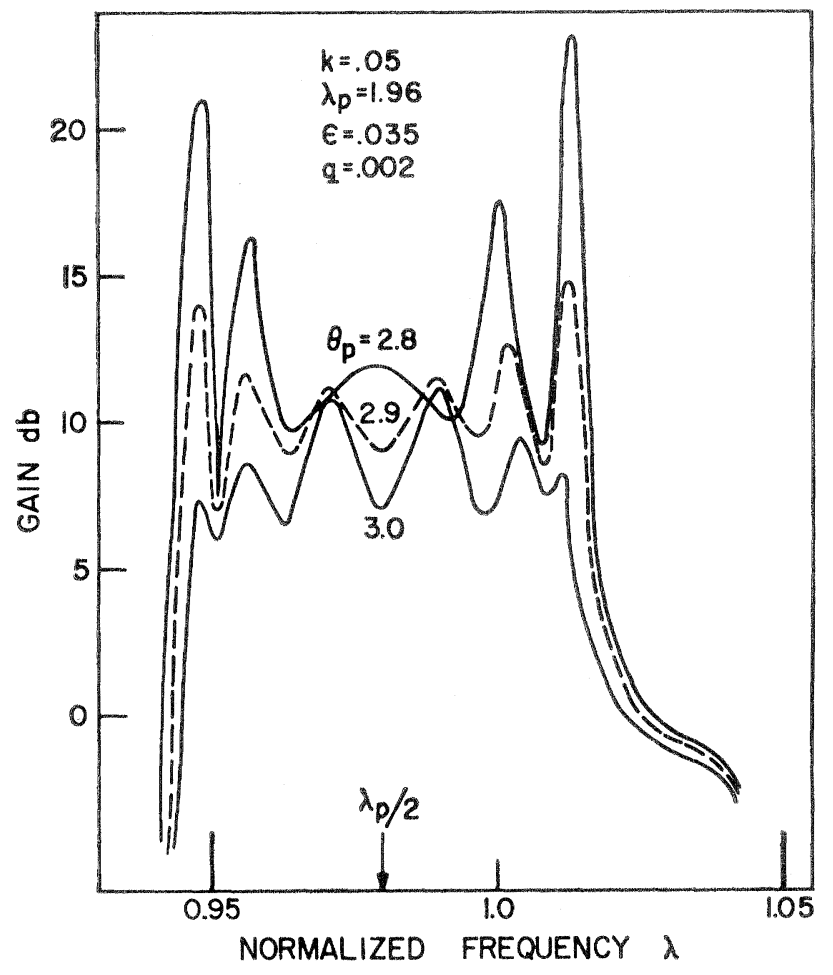

Fig. 8-Gain vs frequency for a six-section amplifier, showing the effect of varying pump phase $\theta_{p}$.

variable capacitance diode), we define the interaction impedance to be

$$
K=\frac{\left|V_{n}\right|^{2}}{2 P}
$$

It is shown in Appendix II that this interaction impedance is a fundamental parameter in all capacitively pumped, traveling-wave parametric amplifiers and should be maximized for strong coupling between the signal and pump frequencies at the diode junction. This also is in agreement with the intuitive idea that for a given power flow the signal voltage should be maximized across the variable capacitance. A similar parameter is important in the design of traveling-wave tubes. For the cold circuit $(\epsilon=0)$,

$$
P=\frac{1}{2} \operatorname{Re}\left[j \omega M i_{n-1} i_{n}{ }^{*}\right],
$$

which can be expressed in terms of voltage as

$$
P=\frac{1}{2} \omega^{3} M C^{2}\left|V_{n}\right|^{2} \sin \theta
$$

using $V_{n-1}=V_{n} e^{j \theta}$. Therefore, neglecting loss,

$$
K=\frac{\sqrt{L / C_{0}}}{\lambda^{3} k \sin \theta} .
$$

Additional physical interpretation of interaction impedance can be obtained by expressing power flow in the form

$$
P=W v_{g}
$$


where $W$ is the total time average stored energy per cavity (half in the electric field, half in the magnetic) for a peak voltage $V_{n}$,

$$
W=\frac{1}{2} C_{o}\left|V_{n}\right|^{2},
$$

and $v_{g}$ is the group velocity,

$$
v_{g}=\frac{\partial \omega}{\partial \theta}=\frac{1}{\sqrt{L C_{o}}} \lambda^{3} k \sin \theta \quad \text { cavities/second. }
$$

Eq. (13) was employed in arriving at (36). Substituting (35) and (36) into the definition of interaction impedance, we again arrive at (33). High impedance results from minimizing electric-energy storage (i.e., decreasing total capacitance $C_{0}$ or physically concentrating all of the electric field at the position of the variable capacitance) and by decreasing the group velocity. The former is a function of the basic cavity design, and the latter is controlled by adjusting the coupling between cavities.

In the vicinity of the lower and upper band-edge frequencies, $\theta$ approaches 0 and $\pi$, respectively, leading to extremely high values of interaction impedance. This results physically from extremely low group velocity. We then have a situation in which the cavities behave somewhat like individual single-cavity amplifiers with coupling predominantly by means of the idler wave. Thus, for example, the gain at midband, which can be attained by increasing the pump amplitude to higher and higher values, is ultimately limited by incipient band-edge instability.

Eq. (33) also indicates that at midband $(\lambda \cong 1$, sin $\theta \cong 1$, the product of interaction impedance and bandwidth $(k)$ is approximately constant. This is related to the bandwidth scaling considerations of Appendix I and is a fundamental result. Since interaction impedance is a measure of gain per section, a basic inverse relationship exists between bandwidth and gain per diode. An analogous impedance-bandwidth relationship obtains in the case of traveling-wave tubes. ${ }^{13}$

The philosophy that was used in determining the input and output impedance matches was to use a simple, physically realizable impedance function with only three adjustable constants. In particular, normalized generator and load impedance functions of the following form were employed:

$$
\begin{gathered}
Z_{o}^{o}=R_{o}^{o}+j X_{o}^{o}=C_{1}+j\left(C_{2} \lambda+\frac{C_{3}}{\lambda}\right), \\
Z_{N+1}^{o}=R_{N+1}^{o}+j X_{N+1}^{o}=C_{4}+j\left(C_{5} \lambda+\frac{C_{6}}{\lambda}\right),
\end{gathered}
$$

corresponding to a series resistor, inductor, and capacitor.

${ }^{13}$ J. R. Pierce, "Traveling-Wave Tubes," D. Van Nostrand Co., Inc., New York, N. Y., Chap. V; 1950.
In all of the curves presented thus far, both $Z_{o}^{o}$ and $Z_{N+1}^{o}$ were chosen so as to constitute a match to the cold filter circuit at midband only; i.e., the normalized input impedance of the coupled cavity circuit is

$$
Z_{\text {in }}^{o}=\lambda k \sin \theta+j \lambda k \cos \theta,
$$

where $\theta$ is the phase shift per section and circuit loss has been neglected. The resistive and reactive parts of (39) are shown by the solid lines in Fig. 10(a). In the calculations corresponding to Figs. 7 through 9, the constants $C_{1} \cdots C_{6}$ were chosen so that the real part of the generator and load impedances exactly matched the resistive part of the cold-circuit impedance only at the center of the pass band $(\lambda=1, \theta=\pi / 2)$. The reactive part was chosen to be zero at midband and to have an approximate conjugate slope to that of the reactance function in (39); i.e., for Figs. 7 through $9, C_{1} \cdots C_{6}$ were adjusted so that

$$
\begin{aligned}
& R_{\theta}^{0}=R_{N+1}^{o}=k, \\
& X_{0}^{o}=X_{N+1}^{o} \cong-\lambda k \cos \theta .
\end{aligned}
$$

These relations are indicated by the dashed curves in Fig. 10(a). The approximate reactive compensation can be effected physically by simply placing a series resonant circuit in series with the transmission line.

The large fluctuations in the gain curves indicate that the above choice of generator and load impedances gives rise to large reflections. Indeed, it is intuitively evident that, at the output, we want to establish a match to the exponentially growing wave. The appropriate output

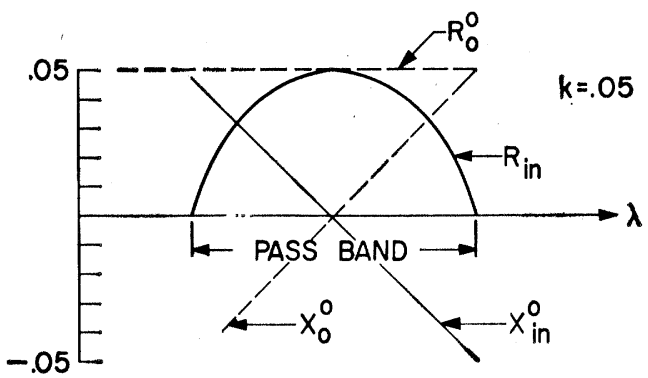

(a)

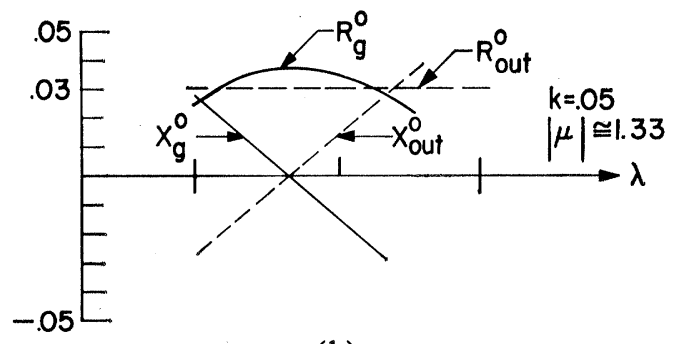

(b)

Fig. 10-The solid curves show the resistance and reactance of: (a) cold circuit input impedance $Z_{\text {in }}^{o}=\lambda k \sin \theta+j \lambda k \cos \theta$; (b) growing wave impedance $Z_{g}^{0}=j \lambda k / \mu_{g}$. In Figs. 7 through 9 , the generator and load impedances were both chosen as indicated by the dashed curves in (a) above; in Fig. 11, the output was matched to the growing wave at two frequencies as indicated by the dashed curves in (b). 
impedance is then

$$
Z_{\text {out }}^{o}=\frac{j \lambda k}{\mu_{g}},
$$

where $\mu_{g}$ is the particular root of the characteristic equation (16) associated with the growing wave. The impedance level at the output is thus reduced below the input level by essentially the voltage gain per cavity. A plot of (41) for a typical case is shown in Fig. 10 (b).

The effect of matching to the growing wave at the output is shown in Fig. 11. With the generator and load impedances matched to the cold circuit [i.e., as in (40)], the curve with large midband gain fluctuations and extremely high peaks at the band edge is obtained; this is the same as one of the curves in Fig. 9. With the output approximately matched to the growing wave [as indicated by the dashed curves in Fig. 10(b)] a greatly smoothed-out response curve results.

Thus, in the absence of stabilizing nonreciprocal attenuation the amplifier response is relatively sensitive to terminating impedances. It is, of course, possible to improve further the results of Fig. 11. Here, the resistive part of the output growing-wave impedance is matched exactly at only two frequencies, as indicated by the magnitude of $R_{N+1}^{o}$ in Fig. 10(b). The use of more com-

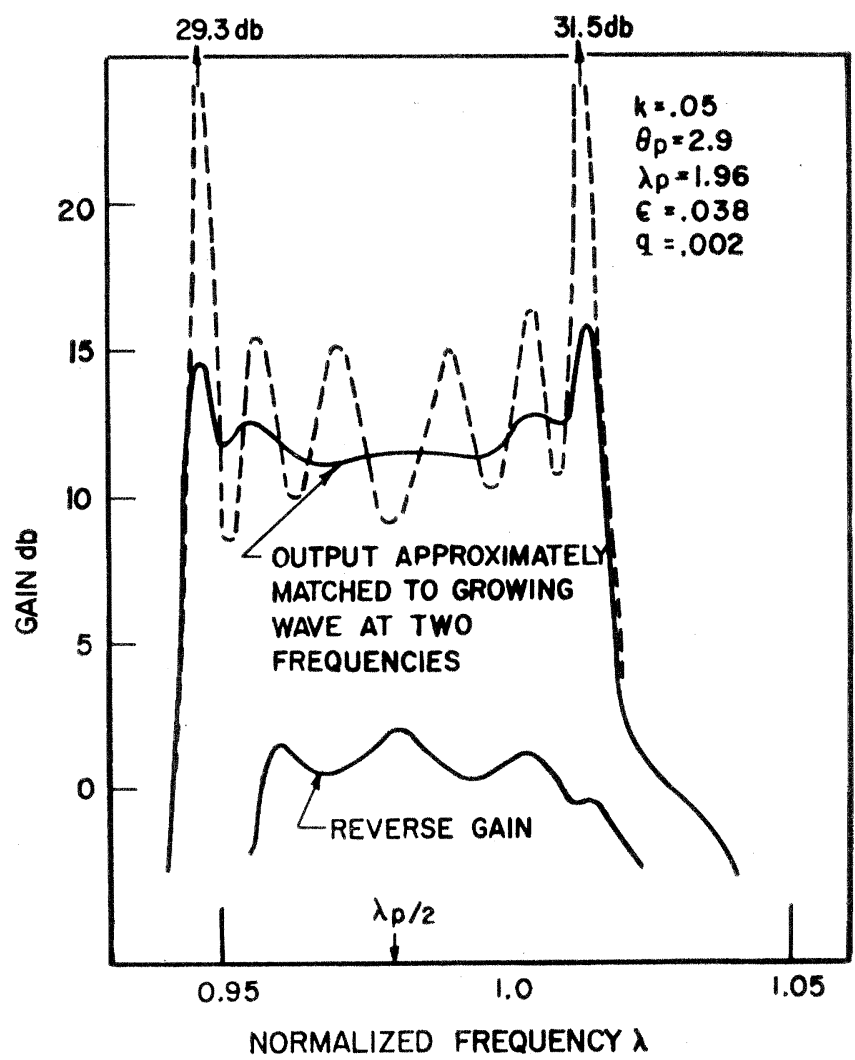

Fig. 11-Effect of matching to the growing wave. The solid gain curve was calculated for an output terminal impedance as shown in Fig. 10(b), which resulted in a smoothed-out frequency response and great reduction in band-edge gain spikes. The extremely low reverse gain shows that unilateral amplification is possible. plicated impedance functions could make the curve of gain vs frequency almost flat. This has been demonstrated experimentally.

Included in Fig. 11 is a curve of reverse gain. Here, the input and output terminals of the amplifier were simply interchanged with the same matching conditions as those described above. It is seen that the reverse gain can be maintained at a value in the neighborhood of unity with 12 - to $15-\mathrm{db}$ gain in the forward direction; essentially unilateral amplification can therefore be achieved.

Midband gain is plotted in Fig. 12 as a function of the number of iterative cavity sections for a particular choice of parameters. The dashed curve represents the level of the exponentially increasing wave alone; this wave predominates after several cavity sections. Notice that an initial excitation loss of about $1 \mathrm{db}$ can be associated with the growing wave for the case shown. In the case of perfect synchronism between the signal and idler waves [i.e., if the phase relation in (17) is satisfied], the growing and decaying waves are excited in phase, each having associated with it $\frac{1}{2}$ the applied voltage. This would lead to an initial growing-wave loss of $6 \mathrm{db}$. With increased departure from synchronism, however, the initial amplitudes of the growing and decaying waves increase (they are no longer excited in phase),

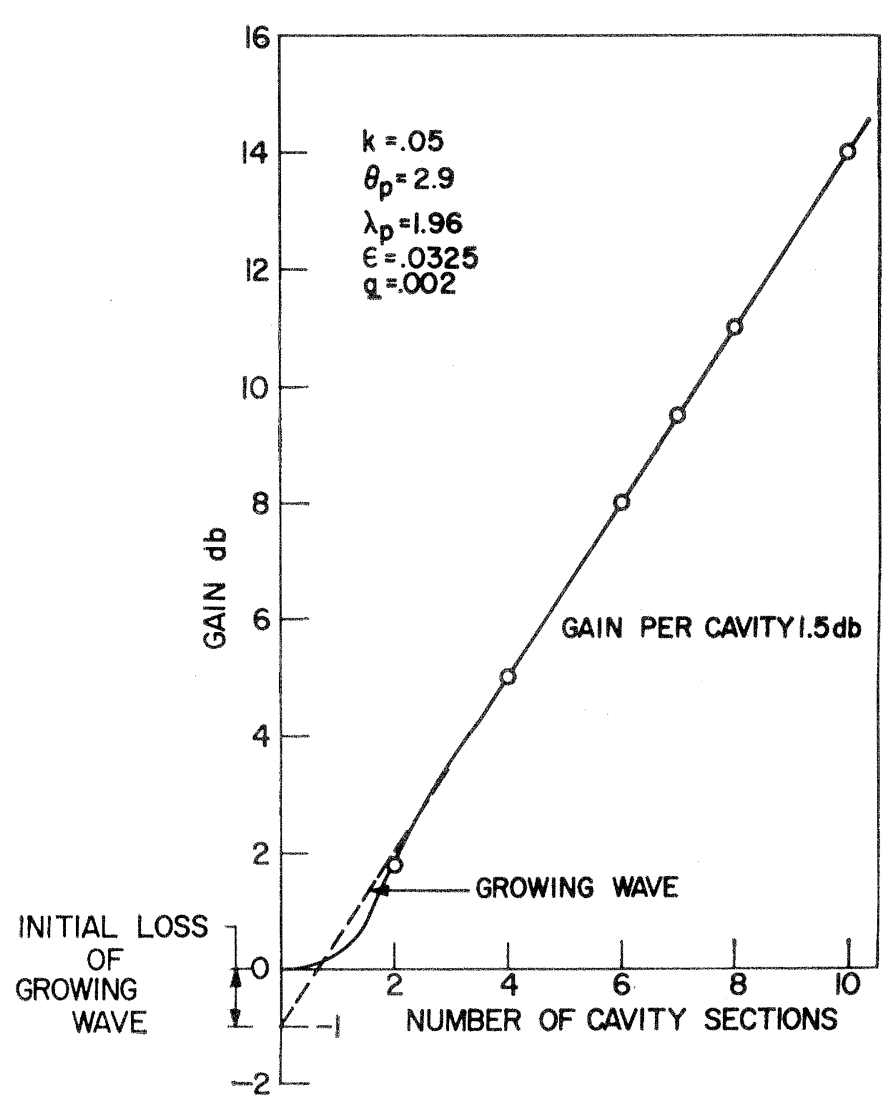

Fig. 12-Gain vs the number of iterated cavity sections as calculated for a particular case. The initial excitation loss of the exponentially growing wave is shown. 
and the initial loss factor of the growing wave decreases, approaching $0 \mathrm{db}$.

The above behavior is, again, entirely analogous to that of traveling-wave tubes and is, in fact, basic to all active coupled-mode systems. In the traveling-wavetube case, there are three characteristic waves (growing, decaying, and unattenuated). With exact synchronism between the electron beam waves and the unperturbed circuit wave, each of the characteristic waves is excited in phase with $\frac{1}{3}$ of the applied voltage, leading to an initial growing-wave loss of $9.54 \mathrm{db}$. This initial loss decreases with departure from synchronism of the unperturbed coupled modes. ${ }^{14}$

Parameter studies of the type described in this section show that the effect of increased circuit loss is twofold; the midband gain is decreased and the response curve is smoothed out, particularly at the band edges. The lowered gain can be made up simply by increasing the pump voltage. It should be noted that the maximum value of $\Delta C / C_{o}$ employed in the calculated results presented in this paper is less than 0.16 , corresponding to a value of $\epsilon$ of 0.04 [see (27)]. Considerably larger capacitance swings are possible and have been achieved experimentally. The improved band-edge response results both from reducing the regenerative effects of reflections where the impedance mismatch is worst and from lowering the $Q$ of the individual cavities in the narrow, bandedge mode of operation. Thus, the response curve of Fig. 11 can be smoothed out still further by the combination of better matching and slight additional circuit loss, although the latter would deteriorate the amplifier noise figure somewhat.

\section{Conclusion}

A class of traveling-wave parametric amplifiers based on coupled-cavity filter circuits has been described. This type of amplifier is particularly suited to microwave frequencies and incorporates new features that overcome some severe difficulties associated with other circuit structures. An analysis has been presented which not only provides detailed information on the operating characteristics of such amplifiers, including the effects of terminal impedances, reflected waves, circuit loss, etc., but also leads to a simple physical picture of the cumulative interaction mechanism based on the coupled-mode concept. This physical picture, presented in terms of a Brillouin diagram, has been emphasized throughout. Fundamental gain and bandwidth considerations have been discussed in terms of an interaction impedance parameter, which is basic to all amplifiers of the traveling-wave type.

Representative calculated curves of gain vs frequency have illustrated the general effects of the various pa-

${ }^{14}$ C. K. Birdsall and G. R. Brewer, "Traveling-wave tube characteristics for finite values of $C$, IRE Trans. ON Electron Devices,
vol. ED-1, pp. 1-11; August, 1954. rameters; these effects have been related to the qualitative description of the amplifier. The calculations have, of course, explored only a restricted range of parameters, but they indicate typical results and, more important, serve as a guide to experimental optimization of pump frequency and phase, terminal impedances, pump strength, etc.

From this investigation, we conclude that unilateral amplification can be obtained over an appreciable portion of the pass band of the coupled-cavity circuit. Stable gains of 12 to $15 \mathrm{db}$ are possible with reasonably flat frequency response. With special stabilization, operation much closer to the ideal synchronous condition would be possible with resulting higher gain per stage. Such stabilization can be obtained, for example, by incorporating nonreciprocal ferrite elements in the coupled-cavity circuit itself or by cascading two or more amplifier sections separated by ferrite isolators and correctly phased with respect to pump voltage. Techniques such as staggered pass band edges and nonuniform phase shift between cavities, which have proved very important in solving various stability problems in traveling-wave tubes, can also be used to advantage; however, these possibilities are not provided in the present analytical approach.

It is not yet known how far bandwidth can be pushed with coupled-cavity amplifiers. Certainly fractional bandwidths of 20 to 30 per cent appear to be possible. As bandwidth increases (i.e., for larger values of the coupling coefficient $k$ ), the Brillouin diagram becomes distorted appreciably from its approximately symmetrical shape at the low values of $k$, and the bandwidth scaling rules presented in Appendix I must be modified. This is an important area for further investigation, and can be most easily accomplished experimentally with the analysis serving as a general guide, as discussed in Part II.

Another area for further study consists of allowing the idler frequency to be several times larger than the signal frequency and to propagate in a separate coupledcavity circuit (or perhaps in a higher pass band). This is important in reducing the noise contribution of the idler channel. With appropriate interpretation, the present theoretical approach can be made to include this situation.

Although noise considerations have not been included explicitly in this treatment, it is shown in Appendix II that over any small frequency region this analysis can be related in simple terms to analyses based on an idealized uniformly distributed model. Therefore, at least to first order, noise performance can be estimated by Tien's results as modified by Shafer ${ }^{15}$ for the case of finite circuit loss.

${ }^{15} \mathrm{C}$. G. Shafer, "Noise figure for a traveling wave parametric amplifier of the coupled-mode type," Proc. IRE, vol. 47, p. 217; December, 1959. 


\section{Appendix I}

\section{BANDWIdTh Scaling}

Throughout the text we have, for convenience, taken $k=0.05$, leading to a 10 per cent cold bandwidth of the filter circuit. We show here that these results can be generalized to other values of $k$ and hence to other bandwidths. The scaling considerations presented here assume $k$ to be small compared with unity. For larger bandwidths, these scaling rules provide at least an approximate guide to the choice of parameters and to the expected performance.

To obtain the basic result, we introduce variables $\delta$ and $\delta_{p}$, respectively:

$$
\lambda_{1}=1+k \delta, \quad \lambda_{p}=2+k \delta_{p} ;
$$

$\delta$ varies from -1 to +1 as $\lambda$ varies from the low-frequency cutoff

$$
\lambda=\frac{1}{\sqrt{1+2|k|}} \cong 1-|k|
$$

to the high frequency cutoff

$$
\lambda=\frac{1}{\sqrt{1+2|k|}} \cong 1+|k| \text {. }
$$

Substituting the above definitions into the determinantal equation (16), we may write

$$
\begin{array}{r}
{\left[2 \delta+\mu+\frac{1}{\mu}-\frac{j q}{k}\right]\left[2\left(\delta-\delta_{p}\right)-\mu e^{j \theta_{p}}+\frac{1}{\mu e^{j \theta_{p}}}-\frac{j q}{k}\right]} \\
+\left(\frac{\epsilon}{k}\right)^{2}=0 .
\end{array}
$$

We see that the values of $\mu$, and hence the gain per resonator, depend only upon the variables, $\delta, \delta_{p}, \theta_{p}$, $q / k$, and $\epsilon / k$. Thus, two amplifiers with different bandwidths (different $k$ values) will have exactly the same gain vs reduced frequency $\delta=\left(\lambda_{1}-1\right) / k$ if they have the same values of $\delta_{p}, \theta_{p}, q / k$, and $\epsilon / k$. In particular, the circuit with the larger bandwidth will require a larger value of the pump parameter $\epsilon$, larger in the ratio of the bandwidths, for the same gain.

This result has been verified by computing gain vs frequency for circuits with different values of $k$ but with identical $\delta_{p}, \theta_{p}, q / k$, and $\epsilon / k$. The result is accurate in detail for small values of $k$; when $k$ becomes as large as 0.1 , some minor quantitative difference arises.

\section{APPENDIX II}

\section{Relation to Tien's Result}

We show here that when the gain per section is small and there is no circuit loss, (16) can be cast in the same form as Tien's result for a uniformly distributed transmission line. First we note that in the absence of the pump $(\epsilon=0)$, each of the brackets of the determinantal equation (16) must vanish separately:

$$
\begin{aligned}
\lambda_{1}{ }^{2}\left[1+2 k \cos \theta_{1}\right] & =1, \\
\lambda_{2}{ }^{2}\left[1+2 k \cos \left(\theta_{2}-\theta_{p}\right)\right] & =1 .
\end{aligned}
$$

Here we have taken $\mu=e^{-j \theta}$ and used the subscripts 1 and 2 for quantities pertaining to the signal and idler frequencies, respectively.

For optimum gain in the presence of the pump, we have shown that $\theta_{1}$ and $\theta_{2}$ should be approximately equal. We therefore write $\theta_{2}=\theta_{1}+\Delta \theta$, where $\Delta \theta$ is assumed to be small. Substituting $e^{-j \theta}$ for $\mu$ with $\theta=\theta_{1}+\Delta \theta / 2+j \Gamma=\theta_{2}-\Delta \theta / 2+j \Gamma$ ( $\Gamma$ small) into (16) and using the definitions of $\theta_{1}$ and $\theta_{2}$ in (44) above, we obtain an approximate equation for $\Gamma$ the growth constant:

$$
\Gamma^{2}=\left(\frac{\xi}{2}\right)^{2}-\left(\frac{\Delta \theta}{2}\right)^{2}
$$

where

$$
\xi^{2}=\frac{\epsilon^{2}}{k^{2} \lambda_{1}{ }^{2} \lambda_{2}{ }^{2} \sin \theta_{1} \sin \left(\theta_{p}-\theta_{2}\right)} .
$$

This has the same form as Tien's equation (41), except that our quantities $\Gamma, \xi$, and $\Delta \theta$ are expressed on a per cavity basis and Tien's are referred to a unit length. From (45) and (46), the gain at midband for $\Delta \theta=0$ is essentially $(\epsilon / k)$ nepers per cavity, which again emphasizes the reciprocal relationship between gain and bandwidth $(k)$.

To bring out further the analogy with Tien's result, we can rewrite our result in terms of the interaction impedance defined earlier [see (30)]. The interaction impedances at signal and idler frequencies are

$$
K_{1}=\frac{\sqrt{L / C_{o}}}{\lambda_{1}{ }^{3} k \sin \theta}, \quad K_{2}=\frac{\sqrt{L / C_{o}}}{\lambda_{2}{ }^{3} k \sin \left(\theta_{p}-\theta_{2}\right)},
$$

and are equal to the square of the voltage appearing across the pump element per unit power flow at signal and idler frequencies, respectively. Therefore, $\xi^{2}$ may be written

$$
\xi^{2}=K_{1} K_{2} \omega_{1} \widetilde{C} \omega_{2} \widetilde{C},
$$

where $\tilde{C} / C_{0}=\tilde{S} / S_{0} \ll 1$ is the fractional change in capacitance. We see that our result is the product of the interaction impedances $K_{1}$ and $K_{2}$ and the variable parts of the susceptances $\omega_{1} \widetilde{C}$ and $\omega_{2} \widetilde{C}$. The gain is clearly increased by increasing any of these quantities.

Tien's expression for $\xi^{2}$, applicable in the case of inductive pumping, can be expressed

$$
\xi^{2}=Y_{01} Y_{02} \omega_{1} L \omega_{2} L
$$

where $Y_{01}$ and $Y_{02}$ are the "interaction admittances" at the signal and idler frequencies, respectively, numerically equal to the square of the current flowing through the pump element per unit power flow $\left(Y_{01}=I_{1}^{2} / 2 P_{1}\right)$, and $\omega_{1} L$ and $\omega_{2} L$ are the variable parts of the reactances added by the pump. In Tien's case, maximum gain is achieved by maximizing any of these quantities; in par- 
ticular, the current flow through the pumped element should be a maximum. Note that for the uniform model considered by Tien, the interaction admittance and the circuit characteristic admittance are equal. The former, however, is a more fundamental parameter in travelingwave parametric amplifiers. Tien's inductive pumping is the dual of our capacitive pumping, and this fact reflects itself in the dual nature of the expressions for $\xi^{2}$.
ACKNOWLEDGMENT

The authors gratefully acknowledge many fruitful discussions with R. D. Weglein and K. P. Grabowski of the Electron Dynamics Department, who contributed greatly to this investigation and carried out the associated experimental program. Thanks are also due B. Fik, who carried out the programming and numerical calculations on an IBM 704 digital computer.

\title{
Coupled-Cavity Traveling-Wave Parametric Amplifiers: Part II-Experiments*
}

\author{
K. P. GRABOWSKI $\dagger$, MEMBER, IRE, AND R. D. WEGLEIN $\dagger$, SENIOR MEMBER, IRE
}

\begin{abstract}
Summary-The filter-circuit approach to broadband travelingwave parametric amplification at microwave frequencies is described from an experimental point of view. The experiment revolves around a series of inductively coupled microwave cavities, each loaded with one variable-capacitance semiconductor diode. Gain-bandwidth products of $2000 \mathrm{Mc}$ with a 350-Mc bandwidth at $S$ band have been obtained by using commercially available diodes. Noise temperatures of $130^{\circ} \mathrm{K}$ have been measured.

Based on the analysis of the companion paper, a qualitative prediction of the gain-frequency behavior is given. The experimental arrangement is set forth in some detail. It consists of a series of these coupled cavities with separate pump power distribution at each diode. Through this flexibility in pump phase shift and amplitude, a variety of advantages is achieved. The effects of the many variables on the performance of the amplifier are described.

Several methods of achieving short-circuit stability of this amplifier are outlined. These are: 1) nonuniform pump phase shift between sections, 2) nonreciprocal loss with ferrites, and 3) nonreciprocal loss with upper pass bands. The experimental behavior of each method is shown, and the noise performance to date is briefly discussed.
\end{abstract}

\section{INTRODUCTION}

$\mathrm{P}$ ARAMETRIC amplification is the result of con $^{-}$ structive mixing of a small signal and a large signal (pump) in a nonlinear reactance. This principle was successfully demonstrated by Hines, ${ }^{1}$ who first employed the voltage-dependent capacitance of a reverse-biased semiconductor diode for this purpose. Since then the principle has been much discussed and applied, and thus the familiar cavity parametric amplifier has evolved. Three frequencies, signal, idler, and

* Received by the IRE, May 5, 1960; revised manuscript received August 19, 1960. Calif.

$\dagger$ Hughes Res. Labs., A Division of Hughes Aircraft Co., Malibu,

${ }^{1}$ M. E. Hines, "Amplification in Nonlinear Reactances Modulators," presented at the 15th Annual Conf. on Electron Tube Res., Berkeley, Calif.; June, 1957. pump, are generally of interest in this device, each of which is usually supported in a resonant circuit or cavity and all are coupled to each other through the nonlinear behavior of the diode capacitor. The high impedance of the resonant circuit at each frequency results in maximum utilization of the signal power to be amplified and in a minimum requirement of pump power for a given capacitance swing. Further consequences of the use of resonant circuits are restricted bandwidth and an approximately constant voltage gain-bandwidth product. Finally, because of its negative resistance character, a circulator is required in the amplifier to make it unilateral and stable.

When a series of these cavity-type amplifiers are properly cascaded, traveling-wave parametric amplification in a filter circuit results. The undesirable features of restricted bandwidth and regeneration largely give way to increased bandwidth and unilateral gain without the use of a circulator, while the advantages of low noise, high gain per diode, and low pump power are retained. The increase in bandwidth is a consequence of large coupling between individual cavities, resulting in a filter-circuit response. Unilateral gain is obtained by properly adjusting the relative phase shift between cavities at the pump frequency. High gain per stage (i.e., per diode) and low pump power are intimately related to the high impedance level of the filter circuit. Additional advantages of this circuit at microwave frequencies are large physical separation of diodes, flexibility in control of higher pass bands, and the ease with which nonreciprocal loss may be incorporated to enhance unilateral gain stability.

It is the object of this paper to describe in detail the filter-circuit approach to traveling-wave parametric amplification, from the experimental point of view, and 